\section{A3.23 VITAMIN D ANTAGONISES THE SUPPRESSIVE EFFECT OF INFLAMMATORY CYTOKINES UPON CTLA-4 EXPRESSION AND REGULATORY FUNCTION}

doi:10.1136/annrheumdis-2013-203216.23

${ }^{1} \mathrm{LE}$ Jeffery, ${ }^{1,2} \mathrm{~K}$ Raza, 'DM Sansom. 'MRC Centre for Immune Regulation, School of Immunity and Infection, Institute of Biomedical Research, University of Birmingham College of Medical and Dental Sciences, Birmingham B15 2TT, UK; 'Department of Rheumatology, Sandwell and West Birmingham Hospitals NHS Trust, Birmingham B18 $70 \mathrm{H}$, UK

Background and Objectives The suppressive protein, cytotoxic T lymphocyte antigen-4 (CTLA-4), is constitutively expressed by TRegs and induced in effector $\mathrm{T}$ cells upon activation. Its crucial role in adaptive immunity is apparent from the fatal autoimmune pathology of CTLA-4 knockout mice and the association of CTLA-4 genetic variants with autoimmunity. We have recently shown that CTLA-4 functions by depleting antigen-presenting cells of their costimulatory ligands, CD80 and CD86 (Qureshi et al, Science 2011). However, little is known about the factors that regulate CTLA-4 expression and function. Since low vitamin D status and elevated Th17 frequencies are evident in many autoimmune conditions, we have investigated the effect of vitamin D and Th17 polarising cytokines upon CTLA-4 expression and function.

Methods Peripheral blood $\mathrm{CD}^{+} \mathrm{CD} 25^{-} \mathrm{T}$ cells were stimulated under Th17 polarising conditions (TGFbeta, IL-1beta, IL- 6 and IL-23) with or without active vitamin $\mathrm{D}\left(1.25(\mathrm{OH})_{2} \mathrm{D}_{3}\right)$. FoxP3 and CTLA-4 were measured by flow cytometry and the vitamin D receptor (VDR) by qPCR. To assess CTLA-4 function, T cells were cultured with anti-CD3 and cells expressing GFP-tagged CD86. CD86-GFP acquisition by T cells with or without CTLA-4 blockade was then monitored by flow cytometry. For suppression assays, separately labelled activated $\mathrm{T}$ cells and $\mathrm{CD} 4{ }^{+} \mathrm{CD} 25^{-}$responder $\mathrm{T}$ cells were co-cultured with dendritic cells in the presence of anti-CD3 and $\mathrm{T}$ cell proliferation assessed at five days by flow cytometry.

Results Vitamin D increased CTLA-4 expression and the frequency of FoxP3+CTLA-4+ T cells. By contrast, Th17 polarising cytokines suppressed CTLA-4. Interestingly, when supplied together, Th17 polarising cytokines synergised with vitamin $D$ resulting in significantly higher CTLA-4 expression than with vitamin D alone. This synergy corresponded with increased VDR expression under Th17 conditions. Using a novel assay to test CTLA-4 function, we further confirmed that these changes in CTLA-4 expression correlated with ligand removal. Moreover, in dendritic cell driven stimulations vitamin D-treated T cell blasts showed enhanced CTLA-4-mediated suppression.

Conclusions Vitamin D overrides the inhibitory effect of proinflammatory Th17 polarising cytokines upon CTLA-4 expression and function. Given the importance of CTLA-4-mediated suppression in the control of autoimmune diseases, including RA, these data highlight the importance of vitamin $\mathrm{D}$ in immune regulation and its potential as a therapeutic agent.

\section{A3.24 ALTERATIONS IN $\gamma \boldsymbol{\delta}$ T CELLS HOMEOSTASIS IN SYSTEMIC SCLEROSIS}

doi:10.1136/annrheumdis-2013-203216.24

TTiago Carvalheiro, ${ }^{2}$ Mariana Santiago, ${ }^{1,3}$ Cláudia Silva, ${ }^{2}$ Maria João Salvador, ${ }^{2} J o s e ́$ António P Silva, ${ }^{1}$ Artur Paiva. ${ }^{1}$ Centro do Sangue e da Transplantação de Coimbra | Instituto Português do Sangue e da Transplantação, IP, Rheumatology Department. Centro Hospitalar e Universitario de Coimbra, Portugal; ${ }^{2}$ Universidade de Aveiro

Background Systemic Sclerosis (SSc) is an autoimmune disease characterised by inflammation and extracellular matrix deposition. There are growing evidences of immune cells alterations, mainly in $\mathrm{T}$ cells subsets, including $\gamma \delta \mathrm{T}$ cells.
Objectives Evaluate the frequency of $\gamma \delta \mathrm{T}$ cells, their distribution among naïve, memory and effector functional compartments, the cytotoxic activity and $\gamma \delta$ repertoire in SSc patients.

Materials and Methods The study enrolled 43 SSc patients, 30 of limited subtype (1SSc) and 13 of diffuse subtype (dSSc). Additionally $14 \%$ of the patients had less than 1 year since diagnosis, $49 \%$ ranging between 1 and 10 years and 37\% with more than 10 years since diagnosis. The healthy control group (HG) included 20 age and gender matched individuals. The phenotypic and functional activity of $\gamma \delta$ T cells was assessed by flow cytometry.

Results It was observed a lower frequency of $\gamma \delta \mathrm{T}$ cells in SSc patients compared to HG. Regarding the cytotoxic activity, it was verified a higher frequency of $\gamma \delta \mathrm{T}$ cells expressing granzime $\mathrm{B}$ and perforin in SSc group, particularly in 1SSc, with a higher $\gamma \delta \mathrm{T}$ cells cytotoxic activity in patients with more than 10 years since the disease onset. Moreover, an increased frequency of $\gamma \delta \mathrm{T}$ cells with a naïve and effector phenotypes was observed in SSc group and these alterations were more evident in dSSc patients. Additionally, patients with pulmonary fibrosis demonstrated an increased frequency of effector memory and naïve $\gamma \delta \mathrm{T}$ cells closely associated to the decrease of central memory cells, when compared to patients without this clinical feature. Patients with more than 10 years since the disease onset, compared to those with less than 1 year since disease diagnosis, presented a higher frequency of effector cells associated to the decrease of central memory cells. Concerning $\gamma \delta$ TCR repertoire assigned by $\mathrm{V} \delta 2$ and $\mathrm{V} \gamma 9$ chains, it was observed in patients with SSc for more than 10 years, an increase of $\mathrm{V} \delta 2^{-} \mathrm{V} \gamma{ }^{-}$ repertoire in naïve, central memory and effector $\gamma \delta \mathrm{T}$ cells when compared to patients with less than 1 year since diagnosis, which exhibit predominantly a $\mathrm{V} \delta 2^{+} \mathrm{V} \gamma 9^{+}$and $\mathrm{V} \delta 2^{+} \mathrm{V} \gamma 9^{-}$repertoire.

Conclusions Important alterations were observed in the frequency, cytotoxic activity, distribution among functional compartments and in $\gamma \delta \mathrm{T}$ cell receptor repertoire in peripheral blood $\gamma \delta$ $T$ cells that seem to be related to the time of disease after diagnosis or to clinical findings.

\section{A3.25 DELETION OF RBP-J IN A MURINE MODEL OF INFLAMMATORY ARTHRITIS REVEALS DIFFERENTIAL PRO- INFLAMMATORY CYTOKINE AND FOXP3 GENE EXPRESSION}

doi:10.1136/annrheumdis-2013-203216.25

1,2Soumya D Chakravarty, ${ }^{2}$ Karmen Au, ${ }^{2}$ Xiaoyu Hu, 1,2Lionel B Ivashkiv. 'Division of Rheumatology, Hospital for Special Surgery; ${ }^{2}$ Department of Medicine, New York Presbyterian Hospital —Weill Cornell Medical Center \& Weill Cornell Medical College of Cornell University, New York, NY

Background/Purpose The DNA-binding protein RBP-J serves as the central transcriptional regulator of the Notch signalling pathway. Prior work done using a knockdown approach of RBP-J in human macrophages and conditional deletion of RBP-J in mouse macrophages has demonstrated diminished LPS-induced expression of TNF $\alpha$, IL-6 and IL-12p40, with IL-1 induction preserved. Elsewhere, it has been observed that host regulatory $T$ cells in RBP-J deficient mice have an attenuated ability to suppress effector $\mathrm{T}$ cell responses in vitro, with augmented proliferation and function of effector $\mathrm{T}$ cells noted in vivo, raising the possibility that dysregulation in the frequency or function of regulatory $T$ cells may contribute to RBP-J's selective modulation of pro-inflammatory mediators. Here, we evaluated the in vivo effects of RBP-J's conditional deletion in the myeloid cell compartment on pro-inflammatory cytokine expression, as well as lymphoid tissue immunocyte composition, using a $\mathrm{K} / \mathrm{BxN}$ serum transfer model of inflammatory arthritis.

Methods RBP-fflox/lox LysM-Cre knock-out (KO) mice with littermate RBP-J ${ }^{+/+}$LysM-Cre controls ( $\mathrm{n}=5$ for each group) were used. After treatment with $\mathrm{K} / \mathrm{BxN}$ serum, the clinical course of arthritis was followed by measuring total joint thickness up to 14 days, at which point the mice were sacrificed. Total joint RNA from each 